\title{
Physiology
}

\section{Foreman in the bone factory}

Etsuko Abe and colleagues have made an unexpected discovery, as they report in Cell (115, 151-162; 2003). They show that thyroidstimulating hormone (TSH) - best known for its role in stimulating the secretion of thyroid hormones in mammals - also regulates the turnover of bone.

During adult life, old bone is continually resorbed and replaced by new bone. These 'remodelling' events must be tightly coupled in both space and time. If, for instance, the activity of bone-resorbing cells (called osteoclasts) exceeds that of bone-depositing cells (osteoblasts), the result is osteoporosis - brittle, weakened bones. The scanning electron micrograph opposite shows the spongy nature of such bones, with many large holes visible (blue). A variety of factors can cause osteoporosis, one of which is high levels of thyroid hormone. Such high levels produce low TSH levels, but it has generally been thought that TSH has nothing to do with the reduction in bone density. Abe et al. now find otherwise.

The authors started by generating mice that lack the cellular receptor that responds to TSH. They found, not surprisingly, that the mice showed little thyroid activity and stunted growth (one of the thyroid's main functions is to ensure proper growth). But the animals also had short, overly light bones. The bones showed severe osteoporosis as well as localized dense patches, suggesting that bone formation and resorption had, to some extent, become spatially separated. Feeding the mice thyroid extracts, to restore their levels of thyroid hormones, increased body weight, but not bone weight or length. This hints that the effects of TSH loss on bone are direct, rather than occurring via thyroid hormones. In support of this, the TSH receptor is found on both osteoclasts and osteoblasts.

Abe et al. also discovered that bone was remodelled much more quickly in the mutant mice than in normal animals, and that more osteoclasts and osteoblasts were formed. These and other findings suggest that TSH usually functions to inhibit both of these cell types. The authors have also begun to define the distinct intracellular signalling

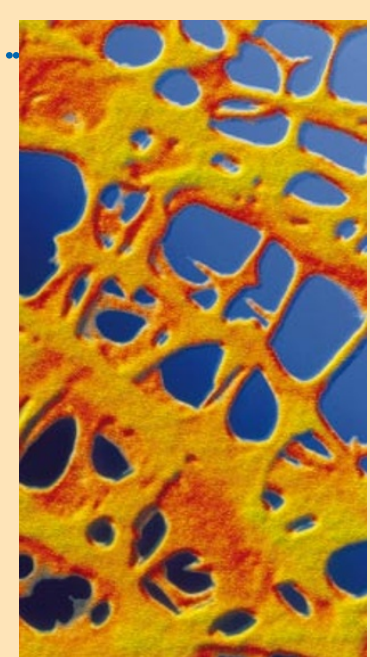

pathways by which TSH prevents the formation and survival of osteoclasts and the formation of osteoblasts. The conclusion is that when TSH is missing, bone remodelling loses a crucial overseer. a new window on this enigmatic source.

Some supermassive black holes in distant galaxies are observed as very bright quasistellar objects, or quasars, that often outshine an entire galaxy of stars. Those black holes accrete (absorb) a lot of gas from their surroundings and in so doing convert a good fraction, say $10 \%$, of the mass energy of that gas to radiation. Their luminosities are nearly equal to the maximum allowed - the so-called Eddington limit, which is proportional to the mass of the object. Sgr A*, in contrast, is extremely dim, radiating at only a billionth of the Eddington limit for its mass. In this respect it resembles the vast majority of black holes in the Universe, which are mostly very dim.

Why is Sgr $\mathrm{A}^{\star}$ so dim? It is true that it has less gas to accrete, compared with the bright black holes in quasars - but this is only around 10,000 times less, not a billion times. Two other factors are believed to contribute to its dimness. First, in contrast to the accretion flows in quasars, the gas flow in Sgr $\mathrm{A}^{\star}$ is radiatively inefficient ${ }^{6}$ : only a very small fraction of its mass energy is converted to radiation. Second, as a direct consequence of this radiative inefficiency, only a small fraction of the available gas actually accretes onto the black hole ${ }^{7}$, the rest being ejected from the system.

This still leaves the fundamental question of exactly how an accretion flow converts mass energy to radiation and why the process is very efficient in bright quasars but highly inefficient in Sgr $\mathrm{A}^{*}$. Something is clearly different about the physics in bright black holes and in dim ones. Unfortunately, the relevant effects are complex and poorly understood, and it has become clear in recent years that real progress will be achieved only when we have more detailed observational clues. The infrared detections of Sgr A* by Genzel et al. ${ }^{4}$, coupled with Chandra's X-ray observations, may be the breakthrough we have been looking for.

The fact that the emission from Sgr $\mathrm{A}^{*}$ varies over tens of minutes, and is almost periodic ${ }^{4}$, indicates that the radiation comes from gas orbiting close to the black hole. This is not unexpected. What is surprising is that $\mathrm{Sgr} \mathrm{A}^{\star}$ emits frequent massive flares of radiation at both infrared and X-ray wavelengths, suggesting that the conversion of mass to radiation is not steady and continuous, but very erratic. There are several possible explanations. One is that the radiatively inefficient accretion flow ejects gas in spurts rather than continuously, and that each ejection of a blob of gas is accompanied by a spurt of radiation ${ }^{8}$. Another is that the amount of gas accreting on to the black hole itself fluctuates, causing the emission to vary ${ }^{9}$. A third idea, perhaps the simplest, is that the accretion engine shorts out once in a while. Lines of magnetic field pervade the accretion disk, and occasionally these may become so distorted that they 'snap' and new lines form. These magnetic reconnection events would produce streams of energetic particles and sparks of radiation ${ }^{10}$ (Fig. 1).

It is not clear at present which, if any, of these ideas is correct, or how the radiation processes actually work in detail. But the beauty of having a flaring source such as Sgr $A^{\star}$ is that each flare provides a new and independent view of the underlying physical processes. So by collecting and studying data on many flares, we may learn much more than from a steady source.
The new discoveries about Sgr $\mathrm{A}^{\star}$ are the direct result of remarkable improvements in technology: the launch of Chandra in 1999, with its superb resolution for X-rays, and the commissioning of adaptive optics on both the Very Large Telescope and the W. M. Keck II Telescope in Hawaii, which allows exquisite observations at infrared wavelengths. A dedicated observational programme using these facilities, and other observatories for radio and sub-millimetre ${ }^{11}$ wavelengths, is clearly the next step. From such observations we will discover whether flares occur simultaneously at all wavelengths (with different amplitudes), or whether the X-ray and infrared flares are largely independent of each other. If the flares are roughly simultaneous, what is the time delay between the emission in different bands? And how much, and in what direction, is the radiation polarized? The answers to these and related questions will doubtless reveal much about the physics of Sgr $\mathrm{A}^{\star}$ and other dim black holes.

Ramesh Narayan is in the Department of Astronomy and the Harvard-Smithsonian Center for Astrophysics, Harvard University, Cambridge, Massachusetts 02138, USA.

e-mail:narayan@cfa.harvard.edu

1. Schödel, R. et al. Nature 419, 694-696 (2003).

2. Ghez, A. M. et al. Astrophys. J. 586, L127-L131 (2003),

3. Baganoff, F. K. et al. Nature $413,45-48$ (2001)

4. Genzel, R. et al. Nature 425, 934-937 (2003).

5. Ghez, A. M. et al. Preprint at <http://arXiv.org/ astro-ph/0309076> (2003).

6. Narayan, R., Yi, I. \& Mahadevan, R. Nature 374, 623-625 (1995).

7. Bower, G. C., Wright, M. C. H., Falcke, H. \& Backer, D. C Astrophys. J. 588, 331-337 (2003).

8. Markoff, S., Falcke, H., Yuan, F. \& Biermann, P. L. Astron. Astrophys. 379, L13-L16 (2001).

9. Liu, S. \& Melia, F. Astrophys. J. 566, L77-L80 (2002). 10. Yuan, E., Quataert, E. \& Narayan, R. Astrophys. J. (in the press). 11.Zhao, J.-H. et al. Astrophys. J. 586, L29-L32 (2003). 